\title{
Correction to: Internal Mammary Sentinel Lymph Node Biopsy in Clinically Axillary Lymph Node-Positive Breast Cancer: Diagnosis and Implications for Patient Management
}

\author{
Peng-Fei Qiu, MD ${ }^{1,2}$, Rong-Rong Zhao, $\mathrm{MD}^{3}$, Wei Wang, $\mathrm{MD}^{4}$, Xiao Sun, $\mathrm{MD}^{2}$, Peng Chen, $\mathrm{MD}^{2}$, \\ Yan-Bing Liu, $\mathrm{MD}^{2}$, Zhi-Guo Liu, $\mathrm{MD}^{5}$, and Yong-Sheng Wang, $\mathrm{MD}, \mathrm{PhD}^{1,2}$ \\ ${ }^{1}$ Shandong Cancer Hospital, Shandong University, Jinan, Shandong, China; ${ }^{2}$ Breast Cancer Center, Shandong Cancer \\ Hospital and Institute, Shandong First Medical University and Shandong Academy of Medical Sciences, Jinan, Shandong, \\ China; ${ }^{3}$ Department of Medicine, Shandong Cancer Hospital and Institute, Shandong First Medical University and \\ Shandong Academy of Medical Sciences, Jinan, Shandong, China; ${ }^{4}$ Department of Radiotherapy, Shandong Cancer \\ Hospital and Institute, Shandong First Medical University and Shandong Academy of Medical Sciences, Jinan, Shandong, \\ China; ${ }^{5}$ Department of Nuclear Medicine, Shandong Cancer Hospital and Institute, Shandong First Medical University and \\ Shandong Academy of Medical Sciences, Jinan, Shandong, China
}

CORRECTION TO: ANN SURG ONCOL

HTTPS://DOI.ORG/10.1245/S10434-019-07705-0

The authors' affiliations are corrected as reflected here.

The original article can be found online at https://doi.org/10.1245/ s10434-019-07705-0.

(C) The Author(s) 2020

Published Online: 9 January 2020

P.-F. Qiu, MD

e-mail: qiupengfei2002@aliyun.com

Y.-S. Wang, MD, PhD

e-mail: wangysh2008@aliyun.com 